\section{Calidad del cuidado y acreditación en centros de hemodiálisis}

\author{
Verónica Guerra-Guerrero*
}

ISSN-PRINT

1794-9831

E-ISSN 2322-7028

Vol. 16 No. 3

Sep - Dic 2019

Cúcuta, Colombia

La enfermedad renal crónica terminal es actualmente considerada un problema de salud pública en el ámbito mundial, por su prevalencia e incidencia creciente en la población, su importancia relativa en la carga de enfermedad del país, su comportamiento crónico o permanente y el requerimiento de una alta complejidad técnica para su manejo (1), en donde enfermería asume un rol fundamental en el cuidado que recibe la población que tiene esta condición. Por lo tanto, la alta complejidad técnica que engloba su tratamiento, es un desafío constante para los cuidados que se otorgan particularmente a los usuarios que se encuentran en la modalidad de hemodiálisis. La enfermedad y tratamiento generan un alto impacto socioeconómico, por lo que también limita o pone en riesgo la vida y calidad de vida de las personas que la padecen, además de afectar el contexto directo que rodea a las personas, como lo es su familia (2).

Derivado de lo anterior, y de las características que presenta el tratamiento en hemodiálisis, los cuidados que se otorgan a las personas bajo esta modalidad, demandan un abordaje integral, en donde el trabajo colaborativo que realizan los profesionales enfermeros, es fundamental para alcanzar los estándares de calidad, que en la actualidad se han instalado en los centros de diálisis a nivel mundial y que a nivel regional se adecuan a condiciones validadas internacionalmente. En este sentido, es primordial reconocer el rol que tiene enfermería, como disciplina que lidera el cuidado en esta población y como responsable de gestionar el cuidado que se otorga desde la especificidad que demanda la terapia dialítica, la cual conlleva enfoques multidisciplinarios, que enfermería debe liderar.

El manejo de la hemodiálisis, que es altamente tecnologizado y complejo, está asociado a la ocurrencia de eventos adversos y a la exposición de riesgos, lo cual puede afectar la calidad y seguridad del cuidado que se otorga a los usuarios con este tratamiento. La calidad y seguridad del cuidado, en la actualidad constituyen criterios que son considerados en la acreditación de los centros de salud $(3,4)$. De este modo, brindar cuidados de calidad en centros de hemodiálisis, es una condición que necesita una preparación especial por parte de los profesionales enfermeros y representa un nivel de complejidad alto, en la que se debe integrar todos los conocimientos y habilidades, con el fin de asegurar el bienestar del usuario y la calidad de los cuidados que se entregan.

El aseguramiento de la calidad de los cuidados, es un aspecto transversal a varias áreas del quehacer profeje común y propio, a través de estándares o criterios de calidad, lo cuales permiten la sistematización de las prestaciones en salud. Al respecto, las enfermeras(os) en el ámbito de hemodiálisis, son responsables de gran parte de las acciones del cuidado, puesto que se encuentran en un lugar privilegiado para cautelar las complicaciones que pueden existir, tomar medidas que reduzcan los riesgos asociados al tratamiento y adoptar estándares de calidad que aseguren el bienestar de los usuarios (5). Es así como la elaboración de listas de chequeo para evaluar la seguridad y calidad de las sesiones de hemodiálisis, por parte de las enfermeras, son aspectos fundamentales que permiten optimizar los cuidados que se otorgan a las personas (6), en consideración a que, aunque existen avances tecnológicos y científicos relevantes en esta área, los pacientes bajo esta modalidad presentan altas tasas de morbimortalidad en forma permanente, por las características que reviste tanto la enfermedad, como su tratamiento. Se describe por ejemplo, que un incremento en el riesgo de muerte y hospitalización, se asocia a niveles de adecuación de diálisis más bajos, incremento en la anemia, niveles de albumina sérica más bajos, y el uso de otros accesos vasculares en vez de la fistula arterio-venosa para hemodiálisis (7). Es por esta razón, que se han desarrollado guías de práctica clínica como la Kidney Disease Outcomes Quality Iniciative (KDOQI) o las European Best Practie Guidelines (EBPG), que derivan en mejorar la calidad del cuidado y los resultados de los pacientes en hemodiálisis.

En base a la existencia de estas guías y de otras, es posible asegurar que las condiciones técnicas del cuidado que se otorga a las personas, deriven en resultados satisfactorios desde la perspectiva biológica o biomédica. Respecto a la perspectiva integral del cuidado, sional en enfermería, en donde se establece un lengua-
Autor de correspondencia

* Doctora en Enfermería. Universidad Catolica de Maule. Correo: Orcid: https://orcid. org/0000-00034517-6678. Talca, Chile. 
es aquí, en donde se encuentra el desafío para los profesionales enfermeros, debido a que estos indicadores de calidad del cuidado en hemodiálisis, no siempre consideran aspectos de la persona como un ser holístico, en donde aspectos como lo ético del cuidado en enfermería son fundamentales (8), así como también lo son, la dimensión psicosocial y espiritual del ser humano. En este sentido, se plantea que los cuidados en hemodiálisis requieren un cuidado por profesionales con un alto nivel de habilidades interpersonales, entre otras, que les permita reconocer las necesidades individuales que son únicas en cada paciente.

En países como Chile (9), los aspectos que se consideran en la acreditación de los centros de diálisis incluyen el respeto a la dignidad de las personas; la gestión de calidad; gestión clínica; acceso, oportunidad y continuidad en atención; competencias del recurso humano; registro; seguridad del equipamiento; seguridad de las instalaciones; y servicios de apoyo. Es en dimensiones como el respeto a la dignidad de las personas, en donde los profesionales de enfermería deben focalizar el desafío de la acreditación de los centros de salud, al igual que en ámbitos de la gestión, debido a que es en donde las acciones lideradas por enfermería permiten la definición de mejora continua y de aspectos relacionados con la seguridad de los pacientes. Similarmente, la gestión clínica es una instancia en donde enfermería tiene la oportunidad de aplicar protocolos y normas actualizadas de las prácticas clínicas, que son relevantes para los pacientes, al mismo tiempo que ofrecen la instancia de que puedan ser evaluadas y mejoradas.

Varias directrices han sido sugeridas para evaluar el cuidado general recibido por los pacientes en el área de salud, y en hemodiálisis, por lo que la calidad de los servicios que se otorgan es una exigencia cada vez mayor y por lo tanto, un desafío permanente para enfermería.

El aumento en la incidencia y prevalencia de la insuficiencia renal crónica, además de los elevados costos derivados del tratamiento de sustitución de la función renal, han llevado a que las fuentes de financiamiento de salud, tanto públicas como privadas, exijan una máxima eficiencia para garantizar una mejor relación entre los recursos utilizados y los resultados obtenidos(10).

Desde esa directriz se torna imperativo que en enfermería se plantee la necesidad permanente de generar un marco de trabajo que permita el desarrollo de métodos y medidas de evaluación del cuidado que se otorga, en donde se plantee el cuidado de los pacientes, con el objetivo de lograr los resultados deseados, según criterios de calidad universal y en donde se privilegie la perspectiva del paciente como un ser humano integral.

\section{Referencias}

1. Rambod M, Rafii F. Perceived social support and quality of life in Iranian hemodialysis patients. J Nurs Scholarsh 2010; 42(3):242-249.

2. Ángel Ángel Zahira Esperanza, Duque Castaño Germán Alberto, Tovar Cortes David Leonardo. Cuidados de enfermería en el paciente con enfermedad renal crónica en hemodiálisis: una revisión sistemática. Enferm Nefrol [Internet]. 2016 Sep [citado 2019 Ago 06] ; 19( 3 ): 202-213. Disponible en: http://scielo.isciii.es/scielo.php?script=sci arttext\&pid=S2254-28842016000300003\&lng=es.

3. Peipert JD, Hays RD. Methodological considerations in using patient reported measures in dialysis clinics. J Patient Rep Outcomes 2017; 1(1):11.

4. Fadili W, Adnouni A, Laouad I. Hemodialysis safety: Evaluation of clinical practice. Saudi J Kidney Dis Transpl 2016; May; 27(3):553-6.

5. de Sousa MR, Silva AE, Bezerra AL, de Freitas JS, Miasso AI. Adverse events in hemodialysis: reports of nursing professionals. Rev Esc Enferm USP 2013; Feb; 47(1):76-83.

6. Fadili W, Adnouni A, Laouad I. Hemodialysis safety: Evaluation of clinical practice. Saudi J Kidney Dis Transpl 2016; May; 27(3):553-6.

7. Grangé S, Hanoy M, Le Roy F, Guerrot D, Godin M. Monitoring of hemodialysis quality-of-care indicators: why is it important? BMC Nephrol 2013; May 24; 14:109.

8. Bennett PN, Neill J. Quality nephrology nursing care: beyond Kt/V. Nephrol Nurs J 2008; JanFeb;35(1):33-7. 
9. Superintendencia de Salud, Gobierno de Chile. Manual del Estándar General de Acreditación para Prestadores Institucionales de Atención Abierta [Internet]. Chile; sf [consultado el 4 de junio de 2019]. Disponible en: http://www.supersalud.gob.cl/observatorio/671/articles-4530_Manual_AA_pdf.pdf

10. Serafim Veras R, Alencar Marinho $C$, Silveira Teófilo T, Alves Silva V, Araújo Moura R. Estándares de indicadores clínicos de calidad en hemodiálisis. J Nurs UFPE [Internet]. 2016 [consultado el 23 de junio de 2019]; 10(7). Disponible en: https://pdfs.semanticscholar.org/ce26/14e70e4c247dade9ecb7b $\underline{5 \mathrm{c} 497815 \mathrm{c} 0554 \mathrm{~b} 0 . \mathrm{pdf}}$ 